\title{
ARTICULACIÓN ENTRE NIVELES EDUCATIVOS: LA EXTENSIÓN UNIVERSITARIA COMO HERRAMIENTA DE INTEGRACIÓN.
}

Ing. Agr. (Esp.) Patricia Verdes ${ }^{(1)}$ y Prof. Julia Adriana Verdes ${ }^{(2)}$

\section{RESUMEN}

Durante los últimos años se ha incrementado notablemente el interés científico en abordar el conocimiento sobre los recursos vegetales autóctonos, con especial énfasis en el desarrollo de biotécnicas para su conservación y/o uso racional. El conocimiento en esta área científica se incrementa en forma exponencial y cada vez es mayor la brecha que existe con los distintos niveles de enseñanza, y consecuentemente con la comunidad en general. El desconocimiento e ignorancia conduce a ideas equívocas. Aún muchos profesionales y docentes, carecen de criterios propios y por ende de una opinión formada, debido al desconocimiento del tema. Se requiere disponer de informaciónformación desde lo formal y desde la educación en sus distintos niveles, a fin de lograr la participación activa de la sociedad mediante la interrelación dinámica entre patrimonio biológico, identidad, conservación, educación y desarrollo regional. Mediante el presente proyecto se pretende acortar esa brecha de conocimientos. La educación ambiental y el respeto por los recursos naturales deben comenzar por nuestro ámbito cotidiano, sumando esfuerzos se puede as- pirar a un cambio global. Además, cuando se conjugan la valorización de la flora autóctona con herramientas biotecnológicas para su conservación y propagación, la puesta en valor de los conocimientos adquiere mayor relevancia. Mediante la formulación y desarrollo del Proyecto de Extensión: Biotecnología y Flora Nativa, patrimonio de todos (UNSL), se procuró acortar esa brecha de conocimientos. En el presente trabajo se comunican las experiencias de articulación y comunicación entre la Universidad y distintos niveles educativos provinciales logradas en el marco del proyecto.

\section{ABSTRACT}

In recent years it has greatly increased interest in addressing scientific knowledge on native plant resources, with special emphasis on the development of biotech for conservation and/or rational use. Scientific knowledge in this area increases exponentially and increasing the gap with the different levels of education is higher, and consequently with the community. The ignorance leads to misconceptions. Even many professionals and teachers, lack of criteria and hence an opinion due to ignorance of the subject. It requires information-formation from

\footnotetext{
(1) Laboratorio de Genética y Biotecnología Vegetal. Departamento de Ciencias Agropecuarias. FICA, UNSL. Ruta Provincial 55 (ex 148 extremo Norte). (5730) Villa Mercedes (San Luis). Cel.: 02657-237164.peverdes@unsl.edu.ar (2) Escuela N ${ }^{\circ} 444$ Jornada Completa "Eva Duarte de Perón” (ex Hogar Escuela Nº 3 Eva Duarte de Perón). 9 de julio 1020. (5730) Villa Mercedes (San Luis).
} 
the formal and from education at different levels in order to ensure the active participation of society through the dynamic interplay between biological resources, identity, conservation, education and regional development. Through this project it aims to bridge that gap of knowledge. Environmental education and respect for natural resources must begin with our daily environment; joining forces can aspire to global change. Also, when the recovery of the native flora with biotechnological tools for conservation and propagation are combined, the enhancement of knowledge becomes more important. Through the formulation and development of the Extension Project: Biotechnology and Native Flora (UNSL) is to bridge that gap of knowledge. In this paper communicate the experiences of articulation and communication between the University and various provincial educational levels achieved in the project.

\section{PALABRAS CLAVES}

Articulación de niveles educativos, Biotecnología, Flora nativa, Educación ambiental.

\section{INTRODUCIÓN}

La provincia de San Luis presenta un gradiente de clima subhúmedo a uno semiárido que determina la bipolarización del paisaje: un sector noreste destinado a actividades agrícolas de secano y uno suroccidental con actividades ganaderas que se intensifican hacia el oeste por disminución de las precipitaciones. Estos paisajes poseen una fisonomía en constante dinámica de cambio debido a diversos factores, entre ellos climáticos, biológicos y socio-económicos.

Así, en los últimos años, el uso del suelo se ha modificado con una extensión hacia el oeste de la frontera agrícola de la provincia de San Luis, en detrimento de las áreas con pastizal natural y bosques nativos (Collado, 1999; Veneciano et al., 2003; Collado y Demaría, 2005). Esta situación se favoreció, entre otros, por los siguientes eventos: el asentamiento de emprendimientos agrícolas de gran magnitud con áreas bajo riego; la aplicación de técnicas de mínima labranza, siembra directa; las perspectivas de estabilidad y rentabilidad que se prevé a corto plazo el mercado de los granos y oleaginosas. Además, en la última década se produjeron registros pluviométricos que han superado a los registros históricos, permitiendo aumentar la probabilidad de éxito en la implantación de pasturas introducidas.

Este avance de la agricultura implica un importante retroceso de las formaciones vegetales relictuales, paisajes fragmentados y la remoción irracional de flora nativa por la fuerte presión de desmonte.

Estos disturbios en la vegetación ocasionan su empobrecimiento tanto en su fisonomía como en su composición florística. Hay pérdida de diversidad de recursos vegetales, animales y microbianos del suelo, invasión por especies exóticas, pérdida de corredores de biodiversidad, erosión eólica e hídrica del suelo, colmatación de lagunas con sedimentos, anegamiento y salinización de suelos (Cisneros, 2002).

Los recursos naturales poseen una peligrosa labilidad potencial con consecuencia grave, irreparable, ante disturbios relativamente leves. Esta vulnerabilidad del ecosistema de pastizales y bosques nativos esta agravada por su escasa representación en los sistemas de áreas protegidas (Bilenca y Miñarro, 2004).

De la misma manera se debe considerar que las plantas nativas ofrecen atributos ornamentales, medicinales, forestales, forrajeros, entre otros, no del todo conocidos 
y por lo tanto no valorizados. Fomentar y dirigir el estudio de las distintas alternativas de protección y manejo de los recursos vegetales constituye una tarea prioritaria de los organismos gubernamentales, de acuerdo al convenio sobre Diversidad Biológica (Naciones Unidas, 1992) ratificado por Argentina (Ley 24.375/94). Este convenio tiene tres objetivos básicos: conservación, utilización sostenible y participación justa y equitativa en los beneficios derivados de los recursos genéticos.

Pero no se pueden valorar, usar y conservar recursos que no se conocen. Además, existe un desconocimiento de tecnologías sencillas, especialmente herramientas biotecnológicas, que contribuyen a la propagación, mejoramiento genético y preservación de flora nativa. Existe escasa valoración de la flora nativa por parte de los consumidores urbanos, la falta de información sobre las alternativas biotecnológicas reproductivas que permitirían una exitosa multiplicación y la tendencia al uso de plantas exóticas en los espacios verdes. La biotecnología ofrece posibilidades que contribuyen marcadamente en los procesos de conservación y puesta en valor de la flora nativa. Resultando una poderosa herramienta para analizar la variabilidad genética, para aumentar el rango de variabilidad disponible, y facilitar la conservación y utilización de los recursos genéticos (Echenique et al., 2010).

En el ámbito productivo, el conocimiento de las plantas nativas cultivadas con fines comerciales presenta una importante alternativa económica de diversificación regional para pequeños productores, permiten el desarrollo de micro-emprendimientos regionales y nacionales, contribuyendo a la conservación y uso racional de dichos recursos.

La educación ambiental es un espacio curricular que permite relacionar distintas áreas del conocimiento, incentivar el interés y aprecio por los recursos naturales, desarrollar nuevas aptitudes y destrezas, y generar cambios actitudinales tanto en los docentes como en los alumnos. $\mathrm{Y}$ en este aspecto cumple un rol importante fortalecer a la articulación entre los distintos niveles educativos, tomando como eje temático a las herramientas biotecnológicas y a la flora nativa.

Bajo este contexto, la implementación del Proyecto de Extensión: Biotecnología y Flora nativa, un patrimonio para todos (Secretaría de Extensión, UNSL), tuvo los siguientes objetivos:

- Impulsar y difundir el conocimiento científico.

- Propender a la valorización y conservación de la flora nativa mediante el uso de la biotecnología.

- Formar un espíritu científico, independencia de criterios y la apropiación crítica de los saberes de esta tecnología.

En el presente trabajo se comunican las actividades y avances logrados en el marco del proyecto de extensión.

\section{METODOLOGÍA}

Para perfeccionar y actualizar a los docentes en su práctica profesional (Ciencias Naturales y Tecnología) y fortalecer la articulación entre los distintos niveles educativos, el Proyecto de Extensión tuvo como destinatarios directos a los docentes y alumnos de los niveles Inicial, primario y secundario, comunicadores sociales y como destinatarios indirectos a la comunidad en general. Con la finalidad de educar y capacitar a la comunidad en el uso sustentable y manejo de la flora nativa, brindar herramientas informativas y formativas, gene- 
rando nuevas prácticas pedagógicas con mayor calidad educativa en distintos niveles de enseñanza, se planificó la organización de cursos de capacitación, talleres y jornadas de difusión científica.

A fin de informar sobre los alcances, beneficios y limitaciones de las aplicaciones biotecnologías en la preservación de recursos vegetales y poner de manifiesto las potencialidades de la flora indígena local, se proyectó la participación en congresos, jornadas y otros eventos de extensión universitaria y educativa, además de la publicación de textos de divulgación científica en medios masivos de comunicación.

Las actividades planificadas se evaluaron con las siguientes metodologías o estrategias: cantidad de docentes y productores asistentes a los talleres y cursos; cantidad y calidad de las propuestas didácticas elaboradas; elaboración de las propuestas didácticas: intencionalidad educativa, selección, organización y graduación de contenidos, estrategias de enseñanza, evaluación. La evaluación del proyecto por parte de los destinatarios: (en los casos que correspondieron) fueron encuestas de evaluación de los eventos organizados.

Los integrantes del equipo de trabajo fueron docentes-investigadores de diferentes formación disciplinar y alumnos de grado de la carrera de Ingeniería Agronómica (FICA, UNSL), y profesoras de nivel primario del Hogar Escuela $\mathrm{N}^{\circ} 3$ Eva Duarte de Perón.

\section{NES \\ RESULTADOS y CONCLUSIO-}

Mediante la implementación de este proyecto se llevaron a cabo las siguientes actividades detalladas en la Tabla 1.

Así, la implementación del presente pro- yecto centró todas las actividades en el eje de conjunción entre Biotecnología y Flora nativa, mediante la transferencia del conocimiento científico y la articulación entre la Universidad y la comunidad educativaproductiva.

Mediante las actividades llevadas a cabo se logró cumplir con la transmisión de información teórica y experiencias sencillas, distintas técnicas y metodologías utilizadas en el laboratorio, que permitieron conceptualizar los procesos biotecnológicos aplicados a la propagación y conservación de los recursos vegetales nativos.

Las innovaciones tecnológicas comunicadas fueron puestas en práctica por los docentes en sus propias escuelas, generando nuevas experiencias pedagógicas y hasta se planteó el desafío de presentarlas en Feria de Ciencias, con resultados exitosos. Asimismo, se motivó a los docentes y alumnos participantes de los talleres y visitas guiadas en el laboratorio de Genética y Biotecnología Vegetal, a trabajar en el laboratorio de sus escuelas o directamente en el aula con elementos sencillos y de fácil adquisición, desarrollando protocolos biotecnológicos simples.

Además, se logró dar a conocer las aplicaciones biotecnológicas que permiten la conservación y el aprovechamiento estratégico de los recursos nativos, disminuyendo el impacto ambiental que provoca la erosión genética por la constante eliminación de flora nativa. La formación de un espíritu científico, independencia de criterios y la apropiación crítica de los saberes de esta tecnología, se logró al informar sobre los alcances y limitaciones de la Biotecnología en todas las actividades llevadas a cabo.

Las instancias previstas de difusión y transferencias de los resultados y avances alcanzados en el proyecto, se lograron me- 
diante la publicación de comunicaciones y resúmenes en diversos eventos científicos nacionales, de índole científico-técnico, educativos y de extensión.

Mediante la transmisión de información con rigor científico se despejaron los temores que generan la innovación científica y la falta de educación biotecnológica básica; temores manifestados en las preguntas e inquietudes de los participantes en las diversas actividades desarrolladas.

De esta manera se cumplieron con las premisas de: - Educar y capacitar a la comunidad productiva en el uso sustentable y manejo de la flora nativa.

- Brindar herramientas informativas y formativas, generando nuevas prácticas pedagógicas con mayor calidad educativa en distintos niveles de enseñanza.

- Perfeccionar y actualizar a los docentes en su práctica profesional (Ciencias Naturales y Tecnología).

- Fortalecer la articulación entre los distintos niveles educativos.

- Informar sobre los alcances, beneficios y limitaciones de las aplicaciones biotecnologías en la preservación de recursos vegetales.

- Poner de manifiesto las potencialidades de la flora indígena local.

En cuanto a los parámetros y estrategias de evaluación y seguimiento de las actividades propuestas, demostraron que existe interés de la comunidad en general por la propagación y la conservación de los recursos vegetales nativos, en especial cuando se emplean biotécnicas. También, quedo manifiesta la inquietud de profundizar el conocimiento en la propagación masiva de diversas especies forestales para la recupera- ción de los ambientes degradados. Por otra parte, surgió el proyecto de diseñar e implementar un espacio verde con ejemplares nativos micropropagados con valor histórico, como centro de recreación y educación ambiental.

Se logró la articulación entre extensión universitaria-docencia-investigación. Mediante la implementación del proyecto resultó muy importante y enriquecedora la transmisión de los conocimientos científicos y tecnológicos adquiridos en el área de Biotecnología a través del desarrollo de la actividad docente y de investigación universitaria (PROICO 50307: Utilización de biotecnologia en la propagación y caracterización genética de especies nativas de la provincia de San Luis. CyT UNSL). La elaboración de las propuestas didácticas, organización y graduación de contenidos, estrategias de enseñanza implementadas, permitió poner de manifiesto la capacidad de formación educativa de los recursos humanos multidisciplinarios del proyecto, para la transmisión de dichos conocimientos a la sociedad; haciendo hincapié en los trabajos desarrollados en el Laboratorio de Genética y Biotecnología Vegetal (FICA, UNSL). Las actividades desarrolladas, tanto en docencia como en investigación, son generadoras de saber científico y tecnológico en el área de Biotecnología dirigida a la conservación, caracterización genética y propagación de especies nativas de la provincia de San Luis. Este conocimiento original surge a partir de recursos humanos formados en múltiples campos disciplinares, por lo tanto resulta muy importante y enriquecedora la transmisión de los mismos a la comunidad en general y específicamente a los docentes de distintos niveles educativos y productores agropecuarios.

Esta articulación con la comunidad, a través de las actividades explicitadas ante- 
riormente, permite hacer extensiva la $\mathrm{ca}^{-}$ pacidad de formación educativa de la universidad hacia la sociedad, cumpliendo con la función social y educativa por las que fue creada. El derecho a una información

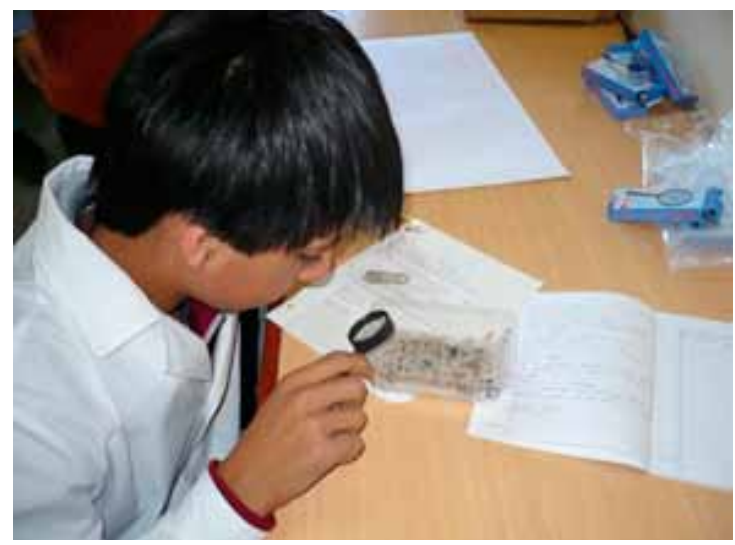

Foto 2. Experiencias sencillas de propagación vegetal: Escuela Especial N 6 "Dra. María Montessori” amplia, detallada y ajustada al rigor de los conocimientos científicos es condición necesaria y suficiente, para ejercer libertad de elección y fomentar el desarrollo de criterios propios.

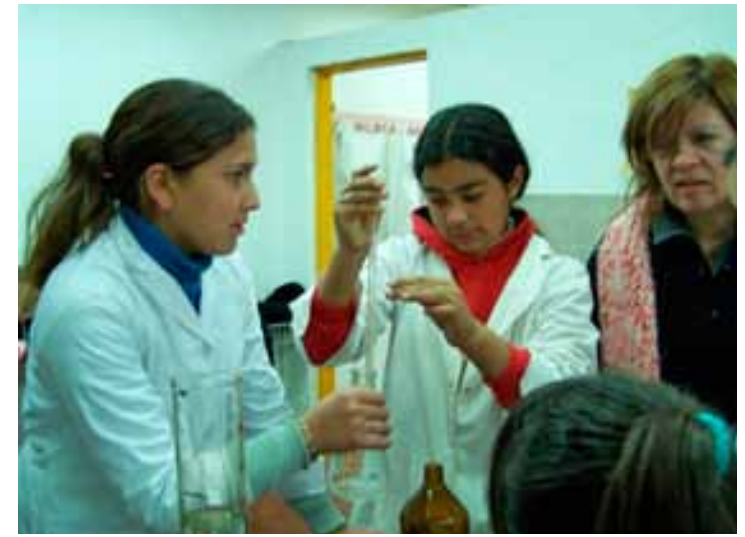

Foto 1. ¿Biotecnología y Flora nativa: aliados para la conservación?. Hogar Escuela Eva Duarte de Perón.

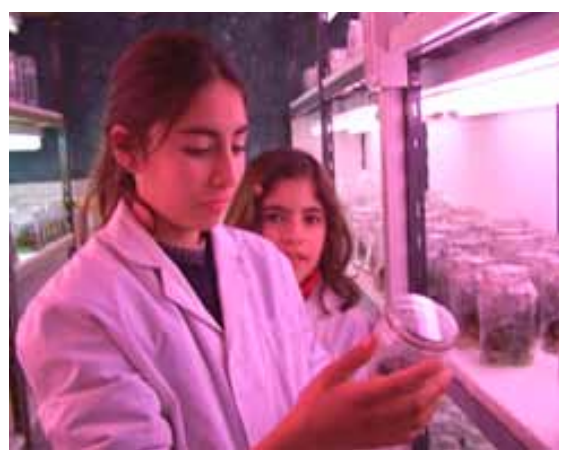

Foto 3. Visitas guiadas en el laboratorio de Cultivo in vitro Sección: nativas.

TABLA 1. Actividades desarrolladas en el marco del Proyecto de Extensión: Biotecnologíay Flora Nativa, patrimonio de todos (Secretaria de Extensión, UNSL).

\begin{tabular}{|c|c|c|c|}
\hline Descripción de la actividad & Fecha & $\begin{array}{l}\text { Lugar de } \\
\text { desarrollo }\end{array}$ & Destinatarios \\
\hline $\begin{array}{l}\text { Organización y coordinación de las Jornadas: } \\
\text { Biotecnología y Flora Nativa. Res. D-717/08. }\end{array}$ & $\begin{array}{c}20-21- \\
22 / 08 / 08\end{array}$ & $\begin{array}{l}\text { Dpto. de Ciencias } \\
\text { Agropecuarias } \\
\text { FICES }\end{array}$ & $\begin{array}{l}\text { Docentes y alumno de } \\
\text { los niveles secundario, } \\
\text { terciario y universitario. } \\
\quad \text { Asistentes: } 45\end{array}$ \\
\hline $\begin{array}{l}\text { Visitas guiadas en el laboratorio de Cultivo in vitro } \\
\text { Sección: nativas. Jornadas: Biotecnología y Flora } \\
\text { Nativa. Res. D-717/08. }\end{array}$ & $\begin{array}{c}20-21- \\
22 / 08 / 08\end{array}$ & $\begin{array}{l}\text { Dpto. de Ciencias } \\
\text { Agropecuarias } \\
\text { FICES }\end{array}$ & $\begin{array}{l}\text { Docentes y alumno de } \\
\text { los niveles secundario, } \\
\text { terciario y universitario. } \\
\text { Asistentes: } 45\end{array}$ \\
\hline $\begin{array}{l}\text { Organización y dictado del Taller teórico-práctico: } \\
\text { ¿Biotecnología y Flora nativa: aliados para la } \\
\text { conservación?". Jornadas: Biotecnología y Flora Nativa. } \\
\text { Res. D-717/08. }\end{array}$ & $21 / 08 / 08$ & $\begin{array}{l}\text { Dpto. de Ciencias } \\
\text { Agropecuarias } \\
\text { FICES }\end{array}$ & $\begin{array}{l}\text { Docentes y alumno de } \\
\text { los niveles secundario, } \\
\text { terciario y universitario. } \\
\text { Asistentes: } 12 \\
\end{array}$ \\
\hline $\begin{array}{l}\text { Organización y dictado del Taller teórico-práctico: } \\
\text { "cAtrapemos el ADN vegetal?". Jornadas: } \\
\text { Biotecnología y Flora Nativa. Res. D-717/08. }\end{array}$ & $\begin{array}{c}20 / 08 / 08 y \\
22 / 08 / 08\end{array}$ & $\begin{array}{l}\text { Dpto. de Ciencias } \\
\text { Agropecuarias } \\
\text { FICES }\end{array}$ & $\begin{array}{l}\text { Docentes y alumno de } \\
\text { los niveles secundario, } \\
\text { terciario y universitario. } \\
\text { Asistentes: } 33\end{array}$ \\
\hline
\end{tabular}




\begin{tabular}{|c|c|c|c|}
\hline $\begin{array}{l}\text { Organización y dictado del Curso de capacitación: } \\
\text { Biotecnología y medio ambiente. CH: } 12 \text { hs. } \\
\text { (http://www.coneiqvillamercedes.com.ar/). } \\
\text { Evaluación integradora final. Res. D-685/08. }\end{array}$ & 08 al 11/08/07 & $\begin{array}{l}\text { XIII CONEIQ8, Congreso } \\
\text { Nacional de Estudiantes } \\
\text { de Ingeniería Química. } \\
\text { FICES-UNSL, Villa } \\
\text { Mercedes (San Luis) } \\
\end{array}$ & $\begin{array}{l}\text { Alumnos universitarios. } \\
\text { Asistentes: } 40\end{array}$ \\
\hline $\begin{array}{l}\text { Asesoramiento y colaboración con la Escuela Especial } \\
\mathbf{N}^{\circ} 6 \text { "Dra. María Montessori", en la elaboración del } \\
\text { proyecto y desarrollo del trabajo experimental sobre el } \\
\text { tema: Domesticación de especies vegetales nativas de la } \\
\text { provincia de San Luis. Feria Nacional de Ciencia y } \\
\text { Tecnología. }\end{array}$ & $29-30 / 09 / 08$ & $\begin{array}{l}\text { Instancia Regional sede } \\
\text { IFDC. Región educativa } \\
\text { 2. Villa Mercedes (San } \\
\text { Luis). }\end{array}$ & $\begin{array}{c}\text { Destinatarios directos: } \\
15 \text { alumnos Segundo } \\
\text { Ciclo. } \\
\text { Destinatarios indirectos: } \\
\text { Docentes y alumnos de } \\
\text { los niveles iniciales, } \\
\text { primario, secundario y } \\
\text { terciario. } \\
\text { Público en general. }\end{array}$ \\
\hline $\begin{array}{l}\text { Implementación y evaluación de la encuesta sobre } \\
\text { percepción pública y biotecnología. }\end{array}$ & $\begin{array}{c}\text { Setiembre a } \\
\text { noviembre/08 }\end{array}$ & Villa Mercedes & $\begin{array}{l}\text { Docentes y alumnos de } \\
\text { los diferentes niveles } \\
\text { educativos; productores } \\
\text { agropecuarios; público } \\
\text { en general. }\end{array}$ \\
\hline $\begin{array}{l}\text { Asistencia a eventos de divulgación y formación } \\
\text { científica-técnico-educativa: } \\
\text { \# XV Jornadas Cuidemos Nuestro Mundo (CNM). } \\
\text { Expositor de póster. } \\
\text { \# VII Jornadas Nacionales de extensión } \\
\text { Universitaria. Expositor de póster. } \\
\text { XXVI Reunión Científica Anual de la Sociedad de } \\
\text { Biología de Cuyo y I Reunión de la Dirección de } \\
\text { Investigación, Ciencia y Técnica (Ministerio de } \\
\text { Salud Gobierno de Mendoza). Expositor de póster. }\end{array}$ & $\begin{array}{l}14-16 / 08 / 08 \\
6-8 / 11 / 08\end{array}$ & $\begin{array}{l}\text { San Luis } \\
\text { San Luis }\end{array}$ & \\
\hline $\begin{array}{l}\text { Publicación de trabajos y textos de divulgación } \\
\text { científica en eventos de comunicación técnico- } \\
\text { educativa: } \\
\text { \# VERDES, P.; VERDES, A.; ROMERO, M.; LARTIGUE, } \\
\text { C. Biotecnología y Flora Nativa, patrimonio para } \\
\text { todos. Exposición de póster y publicación de } \\
\text { resumen. } \\
\text { \# VERDES, P.; NERI, S.; TERENTI, C., LARTIGUE, C. y } \\
\text { ROMERO, M. flores nativas y Biotecnología, una } \\
\text { asociación viable. genéticos. Exposición de póster y } \\
\text { publicación de resumen. }\end{array}$ & $14-16 / 08 / 08$ & $\begin{array}{l}\text { XVI Jornadas Cuidemos } \\
\text { Nuestro Mundo (CNM). } \\
\text { San Luis. }\end{array}$ & 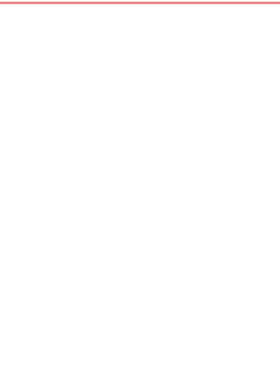 \\
\hline $\begin{array}{l}\text { \# VERDES, P.y VERDES, A. Propuesta de } \\
\text { implementación de un espacio verde con árboles } \\
\text { patrimoniales de la provincia de San Luis. } \\
\text { Exposición de póster y publicación de resumen en } \\
\text { soporte electrónico (CD). ISBN 978-987-1031-73-3. } \\
\text { \# VERDES, P.; LARTIGUE, C.; ROMERO, M. La } \\
\text { educación ambiental como herramienta válida para } \\
\text { la conservación del ambiente. Exposición de póster } \\
\text { y publicación de resumen en soporte electrónico } \\
\text { (CD). ISBN 978-987-1031-73-3. } \\
\text { WERDES, P.; VERDES, A.; DIANA, I. y PEREYRA, M. } \\
\text { Experiencias sencillas pero relevantes en la } \\
\text { domesticación de las especies vegetales nativas de } \\
\text { la provincia de San Luis. Exposición de póster y } \\
\text { publicación de resumen en soporte electrónico } \\
\text { (CD). ISBN } 978-987-1031-73-3 \text {. }\end{array}$ & $6-8 / 11 / 08$ & $\begin{array}{l}\text { VII Jornadas Nacionales } \\
\text { de Extensión } \\
\text { Universitaria. San Luis. }\end{array}$ & $\begin{array}{c}\text { Docentes, } \\
\text { Investigadores, alumnos } \\
\text { de nivel universitario, } \\
\text { terciario, secundario y } \\
\text { primario. } \\
\text { Productores } \\
\text { agropecuarios. } \\
\text { Público en general. }\end{array}$ \\
\hline $\begin{array}{l}\text { \# VERDES, P; LARTIGUE, C; ROMERO, M. } \\
\text { Biotechnology and public perception. Exposición de } \\
\text { póster y publicación de resumen en soporte } \\
\text { electrónico (CD). Publicación impresa en Bjocell (en } \\
\text { prensa). }\end{array}$ & $5-7 / 12 / 08$ & $\begin{array}{l}\text { XXVI Reunión Científica } \\
\text { Anual de la Sociedad de } \\
\text { Biología de Cuyo. } \\
\text { Mendoza. }\end{array}$ & \\
\hline
\end{tabular}

